\title{
SOLUTIONS OF PERIOD SEVEN FOR A LOGISTIC DIFFERENCE EQUATION*
}

\author{
A. BROWN
}

Communicated by James M. Hill

\begin{abstract}
The paper extends earlier results for periodic solutions of the difference equation $w_{n+1}=w_{n}^{2}-A$, where $A$ is a constant and $A>-1 / 4$. Exact equations are given for determining solutions of period seven. The method involves finding roots of two polynomial equations, one of degree 18 and the other of degree 7 . For a given value of $A$, each real root of the equation of degree 18 corresponds to a cyclic solution and the equation of degree 7 gives the seven values of $w_{n}$ in this cyclic solution. The equations are valid whether the periodic solution is stable or unstable, although information about the stability emerges as a by-product. Thus it is possible to tabulate precise intervals of stability in the cases where stable solutions occur.
\end{abstract}

\section{Introduction}

The difference equation

$$
u_{n+1}=2 a u_{n}-b u_{n}^{2}
$$

Received 29 March 1982.

* This paper is based on a talk given at the Australian Mathematical Society Applied Mathematics Conference held in Bundanoon, February 7-11, 1982. Other papers delivered at this Conference appear in Volumes 25 and 26. 
where $a$ and $b$ are positive constants, with $a>1 / 2$, has been used freely in population dynamics as a finite analogue of the logistic differential equation [5, Chapters 2, 3]. Its properties have been discussed in detail in a review article by May [4], who mentions that an equation of this kind is particularly appropriate in populations where the generations are distinct and $u_{n}$ gives the population size in the $n$th generation. Apart from the zero solution $u_{n}=0$, the equation has an equilibrium solution at $u_{n}=(2 a-1) / b$ and for sufficiently large values of $a$ solutions can oscillate about this non-zero equilibrium level. This leads to the possibility of periodic solutions and to questions of the stability of these periodic solutions. The review article by May [4] includes a good deal of information on these matters, not only for equation (1.1) but also for other equations of a similar character.

In examining solutions of equation (1.1) the parameter $b$ is simply a scale factor which can be eliminated by substituting $v_{n}=b u_{n}$. We can take this a step further by using $w_{n}=a-b u_{n}$, which gives the simpler equation

$$
w_{n+1}=w_{n}^{2}-A
$$

with $A=a^{2}-a$. For $a>1 / 2, A$ increases monotonically with $a$ and can be used as an appropriate parameter instead of $a$ in discussing periodic solutions. This was done in a previous paper [1] in establishing equations for periodic solutions with periods $2,3,4,5$ and 6 and the present paper extends these results to solutions with period 7 . The method used is essentially the same and the notation follows that of the previous paper.

For a solution of period 7 , we want to have $w_{n+7}=w_{n}$ for $n=0,1,2, \ldots$ and $i t$ is easy to see that 


$w_{n+2}$ is a polynomial of degree $2^{2}$ in $w_{n}$,
$w_{n+3}$ is a polynomial of degree $2^{3}$ in $w_{n}$,
$\ldots \ldots \ldots \ldots \ldots \ldots \ldots \ldots \ldots \ldots \ldots \ldots \ldots \ldots \ldots \ldots \ldots \ldots \ldots$
$w_{n+7}$ is a polynomial of degree $2^{7}$ in $w_{n}$.

Thus the condition $w_{n+7}=w_{n}$ gives

$$
G_{7}\left(w_{n}\right)=w_{n+7}-w_{n}=0
$$

a polynomial equation of degree 128 in $w_{n}$. Since the condition $w_{n+7}=w_{n}$ is satisfied by the two equilibrium solutions, we can write

$$
G_{7}\left(w_{n}\right)=\left(w_{n}-a\right)\left(w_{n}-1+a\right) H_{7}\left(w_{n}\right)
$$

where $H_{7}$ is a polynomial of degree 126 in $w_{n}$. (The solution $w_{n}=a$ corresponds to $u_{n}=0$ and the solution $w_{n}=1-a$ corresponds to $\left.u_{n}=(2 a-1) / b.\right)$ Now if $b_{1}, b_{2}, b_{3}, \ldots, b_{7}$ are the values of $w_{n}$ for a solution of period 7 , with distinct elements, this solution must contribute a factor

(1.5) $h_{7}(x)=\prod_{i=1}^{7}\left(x-b_{i}\right)=x^{7}-\alpha x^{6}+\beta x^{5}-\gamma x^{4}+\delta x^{3}-\varepsilon x^{2}+\theta x-\zeta$ to the polynomial $H_{7}(x)$ and at most there will be 18 factors of this type. Equation (1.5) defines $\alpha, \beta, \gamma, \delta, \varepsilon, \theta, \zeta$ as symmetrical functions of $b_{1}$ to $b_{7}$ and the functions $\beta, \gamma, \delta, \varepsilon, \theta, \zeta$ can be determined when $\alpha$ and $A$ are specified. If we refer to a solution of this type as a $C 7$ solution, once a suitable value of $\alpha$ is known (for a given $A$ ) the polynomial $h_{7}(x)$ can be constructed and solving $h_{7}(x)=0$ gives the $b_{i}$ values for this $C 7$ solution. The problem of finding suitable values of $\alpha$ is dealt with by showing that $\alpha$ satisfies a polynomial equation of degree 18 .

Section 2 deals with the problem of finding $\beta, \gamma, \delta, \varepsilon, \theta, \zeta$ when $\alpha$ and $A$ are specified and Section 3 establishes the polynomial equation for 
$\alpha$. In obtaining these equations it is assumed that $\alpha^{2}+\alpha-A \neq 0$ and the main problem in Section 4 is to see what modifications are required in the special case where $\alpha^{2}+\alpha-A=0$. Finally, some numerical results are given in Section 5 .

\section{Relationships for $C 7$ solutions}

The equations for the elements $b_{i}$ in a $C_{7}$ solution are

$$
b_{i+1}=b_{i}^{2}-A, i=1,2,3, \ldots, 7,
$$

where $b_{8}=b_{1}$. From the theory of equations, any symmetrical. function of the $b^{\prime} s$ can be expressed in terms of $\alpha, \beta, \gamma, \delta, \varepsilon, \theta, \zeta$ and we try to use equation (2.1) to obtain an alternative form. In doing this it is convenient to introduce the symbol $\Sigma_{0}$ to denote cyclic summation over the indices 1 to 7 (because equations (2.1) have cyclic symmetry). For example, we can write

$$
\alpha^{2}-2 \beta=\Sigma_{0} b_{1}^{2}=\Sigma_{0}\left(b_{2}+A\right)=\alpha+7 A,
$$

since $\sum_{0} b_{2}=\Sigma_{0} b_{1}=\alpha$. Similar working gives

$$
\alpha^{3}-3 \alpha \beta+3 \gamma=\Sigma_{0} b_{1}^{3}=\Sigma_{0} b_{1}\left(b_{2}+A\right)=B_{1}+A \alpha,
$$

where

(2.4) $B_{1}=\Sigma_{0} b_{1} b_{2}=b_{1} b_{2}+b_{2} b_{3}+b_{3} b_{4}+b_{4} b_{5}+b_{5} b_{6}+b_{6} b_{7}+b_{7} b_{1}$.

In the same way,

$$
\begin{gathered}
\text { (2.5) } \alpha^{4}-4 \alpha^{2} \beta+2 \beta^{2}+4 \alpha \gamma-4 \delta=\Sigma_{0} b_{1}^{4}=\Sigma_{0}\left(b_{2}+A\right)^{2}=(\alpha+7 A)+2 A \alpha+7 A^{2}, \\
(2.6) \alpha^{5}-5 \alpha^{3} \beta-5 \alpha \delta+5 \alpha^{2} \gamma+5 \alpha \beta^{2}-5 B \gamma+5 \varepsilon=\Sigma_{0} b_{1}^{5} \\
=\Sigma_{0} b_{1}\left(b_{2}+A\right)^{2}=\Sigma_{0} b_{1}\left(b_{3}+A+2 A b_{2}+A^{2}\right)=\beta_{2}+2 A \beta_{1}+\left(A+A^{2}\right) \alpha,
\end{gathered}
$$

where $\beta_{2}=\Sigma_{0} b_{1} b_{3}$. Note that we can write $\beta=\beta_{1}+\beta_{2}+\beta_{3}$, where $B_{3}=\Sigma_{0} b_{1} b_{4}$. 
Two similar equations which bring in $\theta$ and $\zeta$ are

(2.7) $\alpha^{6}-6 \alpha^{4} \beta+6 \alpha^{3} \gamma-6 \alpha^{2} \delta-12 \alpha \beta \gamma+6 \beta \delta+6 \alpha \varepsilon-6 \theta+9 \alpha^{2} \beta^{2}-2 \beta^{3}+3 \gamma^{2}$

$$
=\Sigma_{0} b_{1}^{6}=\Sigma_{0}\left(b_{2}+A\right)^{3}=B_{1}+4 A \alpha+3 A^{2} \alpha+21 A^{2}+7 A^{3} \text {, }
$$

(2.8) $\alpha^{7}-7 \alpha^{5} \beta+7 \alpha^{4} \gamma-7 \alpha^{3} \delta+14 \alpha^{3} \beta^{2}-21 \alpha^{2} \beta \gamma+7 \alpha^{2} \varepsilon+14 \alpha \beta \delta-7 \alpha \beta^{3}$

$$
\begin{aligned}
+7 \alpha \gamma^{2}-7 \alpha \theta+7 \zeta+ & 7 \beta^{2} \gamma-7 \beta \varepsilon-7 \gamma \delta \\
& =\Sigma_{0} b_{1}^{7}=\gamma_{1}+\left(A+3 A^{2}\right) B_{1}+3 A \beta_{2}+\left(3 A^{2}+A^{3}\right) \alpha,
\end{aligned}
$$

where $\gamma_{1}=\Sigma_{0} b_{1} b_{2} b_{3}$. It will be seen that equations (2.2), (2.3), (2.5), $(2.6),(2.7)$ and (2.8) can be solved to give, in turn, expressions for $\beta, \gamma, \delta, \varepsilon, \theta, \zeta$, although they involve $\beta_{1}, \beta_{2}$ and $\gamma_{1}$ as well as $\alpha$ and $A$. Thus we require equations which allow $\beta_{1}, \beta_{2}$ and $\gamma_{1}$ to be evaluated from $\alpha$ and $A$.

The method used to isolate $\beta_{1}, \beta_{2}$ and $\gamma_{1}$ (described below) involved a fair amount of detailed algebra and it is possible that a shorter method could be devised. As defined by equation (1.5), $\gamma$ and $\delta$ consist of 35 terms while $B$ and $\varepsilon$ have 21 terms. They can be subdivided into groups of 7 terms, each with cyclic symmetry, by writing

$$
\gamma_{2}=\Sigma_{0} b_{1} b_{2} b_{4}, \quad \gamma_{3}=\Sigma_{0} b_{1} b_{2} b_{5}, \quad \gamma_{4}=\Sigma_{0} b_{1} b_{2} b_{6}, \quad \gamma_{5}=\Sigma_{0} b_{1} b_{3} b_{5},
$$

$$
\begin{gathered}
\delta_{1}=\Sigma_{0} b_{1} b_{2} b_{3} b_{4}, \quad \delta_{2}=\Sigma_{0} b_{1} b_{2} b_{3} b_{5}, \delta_{3}=\Sigma_{0} b_{1} b_{2} b_{3} b_{6}, \\
\delta_{4}=\Sigma_{0} b_{1} b_{2} b_{4} b_{5}, \delta_{5}=\Sigma_{0} b_{1} b_{2} b_{4} b_{6},
\end{gathered}
$$

$$
\varepsilon_{1}=\Sigma_{0} b_{1} b_{2} b_{3} b_{4} b_{5}, \quad \varepsilon_{2}=\Sigma_{0} b_{1} b_{2} b_{3} b_{4} b_{6}, \quad \varepsilon_{3}=\Sigma_{0} b_{1} b_{2} b_{3} b_{5} b_{6} .
$$

Then $\gamma=\gamma_{1}+\gamma_{2}+\gamma_{3}+\gamma_{4}+\gamma_{5}, \delta=\delta_{1}+\delta_{2}+\delta_{3}+\delta_{4}+\delta_{5}$, and $\varepsilon=\varepsilon_{1}+\varepsilon_{2}+\varepsilon_{3}$. (The sub-division of $\beta$ has already been described.) If we form the products $\alpha \beta_{i}, \alpha \gamma_{i}, \alpha \delta_{i}$ and $\alpha \varepsilon_{i}$ and make use of equation (2.1), we get a total of 16 equations relating the 16 quantities $\beta_{i}, \gamma_{i}, \delta_{i}$ and $\varepsilon_{i}$ and the solution of these equations gives expressions for $\beta_{1}, \beta_{2}, \beta_{3} ; \gamma_{1}, \ldots, \gamma_{5} ; \delta_{1}, \ldots, \delta_{5} ; \varepsilon_{1}, \varepsilon_{2}, \varepsilon_{3}$. Typical equations are: 
(2.12) $\alpha \beta_{1}=\alpha(1+2 A)+7 A+\beta_{2}+\gamma+\gamma_{1}-\gamma_{5}$,

(2.13) $\alpha \gamma_{2}=A(\alpha+\beta)+\beta_{1}+\gamma_{3}+\gamma_{4}-\delta_{3}+\delta$,

$(2.14) \alpha \delta_{4}=A\left(2 \beta_{1}+\gamma_{2}+2 \gamma_{3}+\gamma_{4}\right)+\gamma_{1}+\gamma_{4}+\delta_{3}+\delta_{5}+\varepsilon_{1}+2 \varepsilon_{3}$,

(2.15) $\alpha \varepsilon_{1}=A\left(7+2 \alpha+2 \beta_{1}+\beta_{3}+2 \gamma_{1}+2 \gamma_{3}+\delta_{1}-\delta_{5}+\delta\right)+\alpha+\beta_{2}+\gamma_{4}+\delta_{3}+\varepsilon_{2}+2 \theta$.

The equations are linear and we can

(i) use the $\alpha \gamma_{i}$ equations to solve for the $\delta^{\prime} s$ (in terms of $\left.\gamma_{i}, B_{i}, \alpha, A\right)$,

(ii) use the $\alpha \delta_{1}, \alpha \delta_{4}$ and $\alpha \delta_{5}$ equations to solve for $\varepsilon_{1}$, $\varepsilon_{2}$ and $\varepsilon_{3}$,

(iii) use the $\alpha \delta_{2}, \alpha \delta_{3}$ and $\alpha \beta_{i}$ equations to solve for the $\gamma^{\prime} \mathrm{s}$,

(iv) use the $\alpha \varepsilon_{i}$ equations to give two equations for $\beta_{1}$ and $B_{3}$

Parts (i) and (ii) of this procedure are straightforward. In part (iii) a key equation is

(2.16) $\left(\alpha^{2}+\alpha-A\right)\left(\gamma_{2}-\gamma_{4}\right)=\left(\alpha^{2}-\alpha-A\right)\left(\beta_{3}-\beta_{2}\right)+2 \alpha\left(\beta_{1}-2 \beta_{2}\right)$

$$
+4 \alpha A+2 \gamma+4 \beta_{1}-2 \beta_{2}+2 \beta_{3} \text {. }
$$

Provided $\alpha^{2}+\alpha-A \neq 0$, this gives $\gamma_{2}-\gamma_{4}$ in terms of $\alpha, A, \beta_{1}, \beta_{2}$, $\beta_{3}$ and we can go on to obtain similar solutions for $\gamma_{i}, \delta_{i}, \varepsilon_{i}$ and $\theta$.

In particular, the solution for $\gamma_{1}$ can be written as

(2.17) $900 r_{1}$

$$
\begin{aligned}
=\left\{3 \alpha^{5}-5 \alpha^{4}-(50+60 A) \alpha^{3}\right. & \left.+(15-250 A) \alpha^{2}+\left(-15-13 A+57 A^{2}\right) \alpha+\left(105 A^{2}-105 A\right)\right\} \\
& +\beta_{1}\left\{30 \alpha^{3}+30 \alpha^{2}-(10+30 A) \alpha-10-96 A\right\} \\
& +\beta_{2}(30 \alpha-18)+\beta_{3}\left\{-30 \alpha^{3}+(60+30 A) \alpha-90-30 A\right\},
\end{aligned}
$$


where $C=\alpha^{2}+\alpha-A$. This solution can be written in different ways, since $\beta_{1}+\beta_{2}+\beta_{3}=\beta=(1 / 2)\left(\alpha^{2}-\alpha-7 A\right)$. For example, the term in $\beta_{2}$ can be eliminated by replacing $\beta_{2}$ by $\beta-\beta_{1}-\beta_{3}$. This is what was done in part (iv) of the procedure to obtain equations relating $B_{1}$ and $B_{3}$. The two equations are

$$
\begin{aligned}
& 0=p_{0}+p_{1} \beta_{1}+p_{3}\left(3 \beta_{3}\right), \\
& 0=q_{0}+q_{1} \beta_{1}+q_{3}\left(3 \beta_{3}\right),
\end{aligned}
$$

with

$$
\begin{aligned}
p_{0}=15 \alpha^{7}+15 \alpha^{6} & -(115+597 A) \alpha^{5}-(635+1200 A) \alpha^{4} \\
& +\left(-1300-2285 A+3165 A^{2}\right) \alpha^{3}+\left(420-1594 A+4875 A^{2}\right) \alpha^{2} \\
& +\left(240+5824 A+3072 A^{2}-2583 A^{3}\right) \alpha+\left(1680 A+588 A^{2}+630 A^{3}\right),
\end{aligned}
$$

(2.21) $p_{1}=45 \alpha^{6}-45 \alpha^{5}-(135+495 A) \alpha^{4}-(195+630 A) \alpha^{3}$

$+\left(1090-675 A+855 A^{2}\right) \alpha^{2}+\left(2840+1620 A+675 A^{2}\right) \alpha$

$$
+\left(2560-792 A+2754 A^{2}-405 A^{3}\right) \text {, }
$$

(2.22) $p_{3}=-15 \alpha^{6}+15 \alpha^{5}+(-155+165 A) \alpha^{4}-(95+270 A) \alpha^{3}$

$+\left(290-55 A-285 A^{2}\right) \alpha^{2}+\left(1160+60 A+255 A^{2}\right) \alpha+\left(376 A-150 A^{2}+135 A^{3}\right)$,

(2.23) $q_{0}=-45 \alpha^{8}-135 \alpha^{7}+(-405+810 A) \alpha^{6}+(-793+1863 A) \alpha^{5}$

$+\left(-1430+4350 A-4320 A^{2}\right) \alpha^{4}+\left(-760+9115 A-8505 A^{2}\right) \alpha^{3}$

$+\left(384+18506 A-14115 A^{2}+6390 A^{3}\right) \alpha^{2}+\left(1536+19432 A-13140 A^{2}+6777 A^{3}\right) \alpha$

$+\left(10752 A-8232 A^{2}+9450 A^{3}-2835 A^{4}\right)$,

(2.24) $q_{1}=135 \alpha^{6}+585 \alpha^{5}+(1275-1485 A) \alpha^{4}+(3015-6210 A) \alpha^{3}$

$+\left(8950-10905 A+2565 A^{2}\right) \alpha^{2}+\left(14120-5940 A+5625 A^{2}\right) \alpha$

$+\left(9472-2856 A+7974 A^{2}-1215 A^{3}\right)$, 
(2.25) $q_{3}=45 \alpha^{6}+195 \alpha^{5}+(625-495 A) \alpha^{4}+(1165-1590 A) \alpha^{3}$

$$
\begin{array}{r}
+\left(2330-3355 A+855 A^{2}\right) \alpha^{2}+\left(2600-2580 A+1395 A^{2}\right) \alpha \\
+\left(2304-1064 A+1890 A^{2}-405 A^{3}\right) .
\end{array}
$$

These equations depend on the assumption that $C \neq 0$. For $p_{1} q_{3}-p_{3} q_{1} \neq 0$, the equations can be solved for $B_{1}$ and $B_{3}$ and then $\beta_{2}=\beta-\beta_{1}-\beta_{3}$.

$O$

Thus for given values of $\alpha$ and $A$, we can evaluate $\beta, p_{0}, p_{1}, p_{3}, q_{0}, q_{1}$, $q_{3}$ and (in general) solve equations (2.18) and (2.19) for $B_{1}$ and $\beta_{3}$. The value of $\beta_{2}$ follows and equation (2.17) gives $\gamma_{1}$. Equations (2.3), $(2.5),(2.6),(2.7)$ and $(2.8)$ can be used to obtain $\gamma, \delta, \varepsilon, \theta$ and $\zeta$ in turn and this defines $h_{7}(x)$. The roots of $h_{7}(x)=0$ correspond to the values of $b_{i}$ for the solution and equation (2.1) can be used to put them in order. The stability of the solution is determined by $\zeta=b_{1} b_{2} b_{3} \ldots b_{7}$, since

$$
\frac{d w_{n+7}}{d w_{n}}=\prod_{i=0}^{6} \frac{d w_{n+i+1}}{d w_{n+i}}=2^{7} \prod_{i=0}^{6} w_{n+i}=2^{7} \zeta
$$

for a $C_{7}$ solution. For local stability, we must have $2^{7}|\zeta|<1$, that is,

$$
-1 / 128<\zeta<1 / 128
$$

\section{Equation for $\alpha$}

To obtain an equation for $\alpha$ we need an additional relationship between $\alpha, \beta, \gamma, \delta, \varepsilon, \theta, \zeta$ and we would like to have it in a form which allows us to eliminate $B_{1}$ and $B_{3}$. Thus a suitable form is an equation similar to (2.18) and (2.19), for we can then write down the equation for $\alpha$ in determinantal form. There are a number of relationships that can be used and the eventual choice was somewhat arbitrary.

One neat-looking set of results comes from eliminating $A$ in equation (2.1). For example, 


$$
b_{3}-b_{2}=\left(b_{2}^{2}-A\right)-\left(b_{1}^{2}-A\right)=b_{2}^{2}-b_{1}^{2}
$$

and hence

$$
\Pi_{0}\left(b_{3}-b_{2}\right)=\Pi_{0}\left(b_{2}^{2}-b_{1}^{2}\right)=\Pi_{0}\left(b_{2}-b_{1}\right) \Pi_{0}\left(b_{2}+b_{1}\right)
$$

where $\pi_{0}$ is used to denote a cyclic product, that is.

$$
\Pi_{0}\left(b_{2}-b_{1}\right)=\left(b_{2}-b_{1}\right)\left(b_{3}-b_{2}\right)\left(b_{4}-b_{3}\right) \ldots\left(b_{1}-b_{7}\right)=\Pi_{0}\left(b_{3}-b_{2}\right) \text {. }
$$

For a $C 7$ solution the $b_{i}$ are distinct and hence $\Pi_{0}\left(b_{2}-b_{1}\right) \neq 0$. Thus equation (3.2) gives

$$
I=\pi_{0}\left(b_{1}+b_{2}\right)
$$

A similar argument leads to

$$
\begin{aligned}
& 1=\Pi_{0}\left(b_{1}+b_{3}\right), \\
& 1=\Pi_{0}\left(b_{1}+b_{4}\right) .
\end{aligned}
$$

When the right-hand side of equation (3.3) is expanded, we can replace $b_{1}^{2}$ by $b_{2}+A$, and so on, until only first powers of each $b_{i}$ are left. This gives (3.6) $1=(\alpha+\beta+\gamma+\delta+\varepsilon+\theta+2 \zeta)+A(7+6 \alpha+5 \beta+4 \gamma+3 \delta+2 \varepsilon)$

$$
+A^{2}(14+9 \alpha+5 \beta+2 \gamma)+A^{3}(7+2 \alpha)
$$

and equations (3.4) and (3.5) give the same result.

Equation (3.6) is clearly a useful equation, for example, for checking the numerical results obtained from the equations in section 2 , but it is less convenient to use it directly to give a relation between $B_{1}$ and $\beta_{3}$. The inconvenience stems from equations (2.7) and (2.8), which involve $\dot{\gamma}^{2}$. There is no difficulty in determining $\theta$ and $\zeta$ numerically from these equations but in the algebraic solution the $\gamma^{2}$ term includes $B_{1}^{2}$ in its expansion and we would prefer linear terms only. (This difficulty can be circumvented but there was no need to to so in this case.)

Another useful set of equations comes from forming the products 
$\alpha \theta, \alpha \varepsilon, \alpha \delta, \ldots, \alpha \beta$. Indeed some of these equations were used in checking the results for $\alpha \beta_{i}, \alpha \gamma_{i}, \alpha \delta_{i}$ and $\alpha \varepsilon_{i}$. Using equation (2.1), these products become

(3.7) $\alpha \beta=3 \gamma+2 \beta+\alpha-\beta_{1}+A(6 \alpha+7)$,

(3.8) $\alpha \gamma=4 \delta+2 \gamma+\beta+\alpha+\gamma_{5}-\gamma_{1}+\beta_{3}+A(5 \beta+5 \alpha+7)$,

(3.9) $\alpha \delta=5 \varepsilon+2 \delta+\gamma+\beta+\alpha+\delta_{5}-\delta_{1}+\gamma_{3}+A\left(4 \gamma+3 \beta+4 \alpha+7+\beta_{1}\right)$,

(3.10) $\alpha \varepsilon=6 \theta+2 \varepsilon+\delta+\gamma+\beta+\alpha-\varepsilon_{1}-\delta_{5}-\gamma_{3}-\gamma_{5}-\beta_{3}$

$+A\left(3 \delta+2 \gamma+2 \beta+3 \alpha+7+\gamma_{1}-\gamma_{5}+\beta_{1}-\beta_{3}\right)$,

(3.11) $\alpha \theta=7 \zeta+\theta+\alpha+\varepsilon_{1}+\delta_{1}+\gamma_{1}+\beta_{1}+A(2 \varepsilon+\delta+\gamma+\beta+2 \alpha+7)$

$+A\left(\delta_{1}-\delta_{5}+\gamma_{1}-\gamma_{3}-\gamma_{5}+\beta_{1}-\beta_{3}\right)$.

From equation (2.2), $\alpha^{2}=2 \beta+\alpha+7 A$ and hence

(3.12) $\alpha(\theta+\varepsilon+\delta+\gamma+\beta+\alpha+1)=7(\zeta+\theta+\varepsilon+\delta+\gamma+\beta+\alpha)+A(2 \varepsilon+4 \delta+7 \gamma+11 \beta+20 \alpha+42)$

$+A\left(\delta_{1}-\delta_{5}+2 \gamma_{1}-\gamma_{3}-2 \gamma_{5}+3 \beta_{1}-2 \beta_{3}\right)$.

It can be shown that

(3.13) $\delta_{1}-\delta_{5}+2 \gamma_{1}-\gamma_{3}-2 \gamma_{5}+3 \beta_{1}-2 \beta_{3}$

$=(1 / 12)\left[\beta_{1}\left(6 \alpha^{2}+22 \alpha+44-18 A\right)-12 \beta_{3}-\alpha^{4}-2 \alpha^{3}+(-3+10 A) \alpha^{2}\right.$ $\left.-(18+22 A) \alpha-126 A-21 A^{2}\right]$,

which means that the final term in equation (3.12) causes no difficulties. What was done, in effect, was to eliminate $\zeta$ between equations (3.6) and (3.12) and replace $\theta, \varepsilon, \delta, \gamma$ and $\beta$ by their expansions in terms of $\alpha, A, \beta_{1}$ and $\beta_{3}$. This gave

$$
0=(2 \alpha-7)\left\{r_{0}+r_{1} \beta_{1}+r_{3}\left(3 \beta_{3}\right)\right\} \text {, }
$$

with

(3.15) $r_{0}=9 \alpha^{8}-72 \alpha^{7}+(6-324 A) \alpha^{6}+(-4+576 A) \alpha^{5}+\left(-347-2424 A+2646 A^{2}\right) \alpha^{4}$

$+\left(476-8432 A+792 A^{2}\right) \alpha^{3}+\left(7404-10108 A+7278 A^{2}-4356 A^{3}\right) \alpha^{2}$

$+\left(6864-3224 A+11772 A^{2}-1296 A^{3}\right) \alpha+\left(-3792 A+4476 A^{2}-4860 A^{3}+2025 A^{4}\right)$, 
(3.16) $r_{1}=8\left(45 \alpha^{5}+75 \alpha^{4}+(33-306 A) \alpha^{3}+(101-552 A) \alpha^{2}\right.$ $\left.+\left(202-72 A+261 A^{2}\right) \alpha+\left(296+30 A+261 A^{2}\right)\right\}$, (3.17) $r_{3}=8\left\{5 \alpha^{4}-34 \alpha^{3}-(53+50 A) \alpha^{2}+(34-6 A) \alpha+\left(72+14 A+45 A^{2}\right)\right\}$.

The factor $2 \alpha-7$ in equation (3.14) corresponds to the case where $b_{i}=(1 / 2)$ for $i=1,2, \ldots, 7$. This is an equilibrium solution corresponding to $a=1 / 2$ and $A=1 / 4$ and it is easy to check that equations (3.3), (3.4), (3.5) and (3.12) are satisfied in this case. Thus we can expect it to emerge as a possible solution of the equations but since we have stipulated that $a>1 / 2$ we can ignore this solution. This leaves

$$
0=r_{0}+r_{1} \beta_{1}+r_{3}\left(3 \beta_{3}\right) .
$$

As with equations (2.18) and (2.19), equation (3.18) depends on the condition that $C=\alpha^{2}+\alpha-A \neq 0$. For the three equations to be consistent, we must have

$$
0=E(\alpha, A)=\left|\begin{array}{lll}
p_{0} & p_{1} & p_{3} \\
q_{0} & q_{1} & q_{3} \\
r_{0} & r_{1} & r_{3}
\end{array}\right|
$$

and this is (almost) the equation we want for $\alpha$. An examination of the leading terms shows that $E(\alpha, A)$ is a polynomial of degree 20 in $\alpha$ rather than a polynomial of degree 18 . Numerical checks gave $E(0,0)=0=E(-1,0)$ and $E(1,2)=0=E(-2,2)$, which suggests that $\alpha^{2}+\alpha-A$ might be a factor and this was verified by expressing $p_{0}$ as

$$
p_{0}=c p_{0}^{(1)}+p_{0}^{(2)}
$$

where $p_{0}^{(2)}$ is linear in $\alpha$. Similar expressions were obtained for $p_{1}, p_{3} ; q_{0}, q_{1}, q_{3} ; r_{0}, r_{1}, r_{3}$ and the determinant obtained from the remainder terms, that is, 


$$
\left|\begin{array}{lll}
p_{0}^{(2)} & p_{1}^{(2)} & p_{3}^{(2)} \\
q_{0}^{(2)} & q_{1}^{(2)} & q_{3}^{(2)} \\
r_{0}^{(2)} & r_{1}^{(2)} & r_{3}^{(2)}
\end{array}\right|
$$

proved to have a factor $C$. With this assurance that $C$ is a factor of $E(\alpha, A)$ brute force was used to expand $E(\alpha, A)$ and write it in the form

$$
E(\alpha, A)=(36450)\left(\alpha^{2}+\alpha-A\right) D(\alpha, A),
$$

where

$$
D(\alpha, A)=\sum_{n=0}^{\perp 8} k_{n} \alpha^{n},
$$

with

$$
\text { (3.23) } \begin{aligned}
k_{18} & =k_{17}=1, k_{16}=4-57 A, k_{15}=20-136 A, \\
k_{14} & =110-380 A+1188 A^{2}, k_{13}=638-1048 A+3740 A^{2}, \\
k_{12} & =3828-2054 A+9652 A^{2}-11924 A^{3}, \\
k_{11} & =10452-824 A+23660 A^{2}-42168 A^{3}, \\
k_{10} & =27225-42796 A+53404 A^{2}-100588 A^{3}+61950 A^{4}, \\
k_{9} & =60665-113352 A-22652 A^{2}-187504 A^{3}+228070 A^{4}, \\
k_{8} & =120032-272825 A+186604 A^{2}-468700 A^{3} \\
k_{7} & =195632-602304 A+439044 A^{2}+148168 A^{3} \\
k_{6} & =494368-1254960 A+311856 A^{2}+381644 A^{3}+1402822 A^{4}-1211764 A^{5}+254932 A^{6},
\end{aligned}
$$




$$
\begin{aligned}
& k_{5}=886384-389280 A-1462000 A^{2}+477600 A^{3} \\
& +644702 A^{4}-1176536 A^{5}+638876 A^{6} \text {, } \\
& k_{4}=698944+1180624 A+110448 A^{2}-2616528 A^{3} \\
& +2721088 A^{4}-3248366 A^{5}+1558332 A^{6}-217188 A^{7}, \\
& k_{3}=-424704+976896 A+2347360 A^{2}-3500032 A^{3} \\
& +4361008 A^{4}-3148288 A^{5}+1377620 A^{6}-376200 A^{7}, \\
& k_{2}=-566272-2844416 A+6158720 A^{2}-9309792 A^{3}+9790784 A \\
& -6466416 A^{5}+3379216 A^{6}-997668 A^{7}+97929 A^{8}, \\
& k_{1}=573440-3377152 A+5289728 A^{2}-7828480 A^{3}+6725680 A^{4} \\
& -4708576 A^{5}+2193936 A^{6}-573984 A^{7}+86913 A^{8} \text {, } \\
& k_{0}=1048576-2277376 A+4746240 A^{2}-5809408 A^{3}+6054976 A^{4} \\
& -4572528 A^{5}+2629296 A^{6}-1116432 A^{7}+251424 A^{8}-18225 A^{9} .
\end{aligned}
$$

For a given value of $A$ the coefficients $k_{0}$ to $k_{18}$ can be evaluated and the appropriate values of $\alpha$ are the real roots of $D(\alpha, A)=0$.

\section{Special case $C=0$}

It might appear that the special case $\alpha=0$ requires attention because of the use of equations such as (2.12) to (2.15) or (3.7) to (3.11). However when a check was made this case produced no difficulties. For example, in the derivation of equation (2.12),

$$
\begin{aligned}
\alpha \beta_{1} & =\left(\Sigma_{0} b_{1}\right)\left(\Sigma_{0} b_{1} b_{2}\right)=\Sigma_{0}\left\{b_{1}\left(b_{1} b_{2}+b_{2} b_{3}+b_{3} b_{4}+b_{4} b_{5}+\ldots b_{7} b_{1}\right)\right\} \\
& =\Sigma_{0} b_{1}^{2}\left(b_{2}+b_{7}\right)+\Sigma_{0}\left(b_{1} b_{2} b_{3}+b_{3} b_{4} b_{1}+b_{4} b_{5} b_{1}+b_{5} b_{6} b_{1}+b_{6} b_{1} b_{1}\right) \\
& =\Sigma_{0}\left(b_{2}+A\right)\left(b_{2}+b_{7}\right)+\gamma_{1}+\gamma_{4}+\gamma_{3}+\gamma_{2}+\gamma_{1} \\
& =(\alpha+7 A)+2 A \alpha+\beta_{2}+\gamma+\gamma_{1}-\gamma_{5} .
\end{aligned}
$$

This expansion is still valid when $\Sigma_{0} b_{1}=0$; the equation reduces to $0=7 A+\beta_{2}+\gamma+\gamma_{1}-\gamma_{5}$. Checking the details in this way gave $k_{0}=0$ as the equation for $A$, in agreement with $D(0, A)=0$. 
When $C=0$, equation (2.16) is still valid but it no longer gives an equation for $\gamma_{2}-\gamma_{4}$ and the solution for the $\gamma_{i}$ breaks down. This invalidates most of the subsequent equations in Section 2 and it is better to make a fresh start, replacing $\alpha^{2}$ by $A-\alpha$ wherever it occurs. Equations (2.2), (2.5) and $(2.6)$ become

$$
\beta=-\alpha-3 A, \quad 3 \gamma=\alpha(2-9 A)-2 A+B_{1},
$$

$$
\begin{aligned}
3 \delta & =\alpha(7 A-2)+9 A^{2}-4 A+\alpha \beta_{1}, \\
15 \varepsilon & =\alpha\left(7-41 A+45 A^{2}\right)+\left(21 A^{2}-7 A\right)+3 \beta_{2}-(5 \alpha+9 A) \beta_{1} .
\end{aligned}
$$

The equations for $\alpha \beta_{i}, \alpha \gamma_{i}, \alpha \delta_{i}$ and $\alpha \varepsilon_{i}$ are still valid, so equation (2.16) can be used; hence

$$
0=2 \alpha\left(\beta_{2}-\beta_{3}\right)+2 \alpha\left(\beta_{1}-2 \beta_{2}\right)+4 \alpha A+2 \gamma+4 \beta_{1}-2 \beta_{2}+2 \beta_{3} .
$$

If we replace $\gamma$ From equation (4.1) and put $\beta_{3}=\beta-\beta_{1}-\beta_{2}$, we get

$$
3 \beta_{2}=(3 \alpha+2) \beta_{1}+(3 A-2) \alpha-4 A \text {, }
$$

and from this

$$
3 \beta_{3}=-(3 \alpha+5) \beta_{1}-(3 A+1) \alpha-5 A \text {. }
$$

Thus we can put $\beta_{2}$ and $\beta_{3}$ in terms of $\beta_{1}, \alpha, A$ whenever they appear.

From the $\alpha \beta_{1}$ and $\alpha \beta_{3}$ equations it is straightforward to obtain

$$
\begin{aligned}
& \gamma_{5}=\gamma_{1}+\beta_{1}+\alpha+5 A, \\
& \gamma_{3}=\gamma_{1}+(1 / 3)\left\{\beta_{1}(2-2 \alpha-3 A)+(A+2) \alpha+10 A-3 A^{2}\right\},
\end{aligned}
$$

and hence $\gamma_{2}+\gamma_{4}=\gamma-\gamma_{1}-\gamma_{3}-\gamma_{5}$ can also be expressed in terms of $\gamma_{1}, \beta_{1}, \alpha, A$. The main problem is to find an equation for $\gamma_{2}-\gamma_{4}$ as a replacement for equation (2.16).

An unexpected bonus came from the equation for $\alpha \delta_{3}$, which is

$$
\alpha \delta_{3}=A\left(\alpha+\beta+\gamma-\beta_{1}-\gamma_{2}\right)+\varepsilon+\delta_{1}+\delta_{5}+\gamma_{5}+\beta_{2} .
$$


As mentioned in Section 2, it is easy to solve for the $\delta^{\prime} \mathrm{s}$ in terms of $\gamma_{i}, \beta_{i}, \alpha, A$ and these solutions were substituted for $\delta_{1}, \delta_{3}$ and $\delta_{5}$ in equation (4.6). The results in equations (4.1), (4.3), (4.4) and (4.5) were used to obtain an equation for $\beta_{1}$ and this gave

$$
\beta_{1}(4-3 A+\alpha)=3 A^{2}-3 A-4 \alpha A \text {, }
$$

a surprisingly neat result. Note that if the co-factor of $\beta_{1}$ is zero in this equation, then the right-hand side of the equation must be zero also and we either have

$$
3 A=4+\alpha \text {, with } A=0 \text {, }
$$

or

$$
3 A=4+\alpha \text {, with } 3 A=4 \alpha+3:
$$

Neither of these is compatible with the assumption that $C=0$. Thus we can take $Q=4-3 A+\alpha \neq 0$ and regard equation (4.7) as an equation for $B_{1}$, in terms of $\alpha$ and $A$. From equations (4.3), (4.4) and (4.1),

$$
\begin{aligned}
& Q B_{2}=3 A^{2}-8 A+(2 A-2) \alpha, Q B_{3}=3 A^{2}-2 A+(2 A-1) \alpha, \\
& Q \gamma=-3 A+\left(2-13 A+9 A^{2}\right) \alpha, \\
& Q \delta=-6 A+17 A^{2}-9 A^{3}+\left(-2+8 A-3 A^{2}\right) \alpha, \\
& Q \varepsilon=-3 A+8 A^{2}-3 A^{3}+\left(1-10 A+20 A^{2}-9 A^{3}\right) \alpha .
\end{aligned}
$$

To obtain similar equations for $\gamma_{1}$ to $\gamma_{5}$, the equations used were

$$
\begin{aligned}
& \beta_{1}^{2}=7 A^{2}+4 A \alpha+\beta_{1}+2 A \beta_{2}+2 \beta_{3}+2\left(\delta_{1}+\delta_{4}\right), \\
& \beta_{2}^{2}=7 A^{2}+2 A \alpha+\beta_{2}+2 A \beta_{3}+2 \gamma_{3}+2\left(\delta_{1}+\delta_{5}\right), \\
& \beta_{3}^{2}=7 A^{2}+2 A \alpha+\beta_{3}+2 A \beta_{1}+2 \gamma_{4}+2\left(\delta_{4}+\delta_{5}\right) .
\end{aligned}
$$

These equations are valid in the general case and the $\beta_{1}^{2}$ equation can be used in evaluating $\gamma^{2}$, which occurs in equations (2.7) and (2.8). If we 
take $\beta_{2}^{2}-\beta_{3}^{2}$ and substitute for $\delta_{1}$ and $\delta_{4}$, the resulting equation is (4.10) $\beta_{2}^{2}-\beta_{3}^{2}=2\left(\beta_{2}-\beta_{3}\right)+\alpha\left(\gamma_{1}-\gamma_{3}\right)+\left(2 \gamma_{3}+\gamma_{5}-\gamma_{2}-\gamma_{4}\right)$ $-(1+A) \alpha-7 A+A\left(4 \beta_{3}-3 \beta_{1}-\beta_{2}\right)$.

The essential point about this equation is that $\gamma_{3}, \gamma_{5}$ and $\gamma_{2}+\gamma_{4}$ can be expressed in terms of $\gamma_{1}$ (plus terms in $\alpha, A, B_{i}$ ) and in the special case $C=0$ this leads to

$$
2 Q^{2} \Upsilon_{1}=-44 A+72 A^{2}-30 A^{3}+\left(-8+23 A-40 A^{2}+18 A^{3}\right) \alpha \text {. }
$$

Similar expressions for $2 Q^{2} \gamma_{3}, 2 Q^{2} \gamma_{5}$ and $2 Q^{2}\left(\gamma_{2}+\gamma_{4}\right)$ follow immediately and the $\beta_{1}^{2}$ equation gives $2 Q^{2}\left(\gamma_{2}-\gamma_{4}\right)$. This gives a set of equations for the $\gamma_{i}$ and solutions for $\delta_{i}, \varepsilon_{i}$ follow in a straightforward way.

Equations (2.7) and (2.8) gave

$$
\text { (4.12) } \begin{aligned}
& 2 Q^{2} \theta=8 A+56 A^{2}-116 A^{3}+76 A^{4}-18 A^{5}+\left(-4+47 A-78 A^{2}+44 A^{3}-6 A^{4}\right) \alpha, \\
& \text { (4.13) } 14 Q^{2} \zeta=-54 A+505 A^{2}-690 A^{3}+308 A^{4}-42 A^{5} \\
&+\left(2-76 A+586 A^{2}-922 A^{3}+574 A^{4}-126 A^{5}\right) \alpha
\end{aligned}
$$

and equation (3.11) was used as a check for these expressions. Thus for given values of $\alpha$ and $A$, if it turns out that $C$ is zero or close to zero we have to switch from the equations in Section 2 and instead use equations $(4.7),(4.8)$ or $(4.1),(4.12)$ and (4.13) to calculate the coefficients in the polynomial $h_{7}(x)$. As before, the roots of $h_{7}(x)=0$ give the elements of the $C 7$ solution and the value of $\zeta$ tells whether the cycle is stable or unstable.

We also have to check on the equation for $\alpha$ in the case when $C=0$. When the expressions obtained for $B, \gamma, \delta, \varepsilon, \theta$ and $\zeta$ were substituted in equation (3.6) it was found that the condition

$$
\text { (4.14) } \quad 0=224-508 A+404 A^{2}-104 A^{3}+\left(80-107 A-10 A^{2}+24 A^{3}\right) \alpha
$$

had to be satisfied for equation (3.6) to hold. Another equation that is available in the general case is 


$$
\alpha \zeta=\alpha+7 A+A\left(\theta+\varepsilon_{1}+\delta_{1}+\gamma_{1}+\beta_{1}+\alpha\right),
$$

which is similar to equations (3.7) to (3.11), and this equation was also used as a check equation in the case $C=0$. This gave a somewhat different condition

(4.16) $0=224-380 A+188 A^{2}-24 A^{3}+\left(208-435 A+310 A^{2}-72 A^{3}\right) \alpha$.

Equations (4.14) and (4.16) can obviously be combined in different ways to obtain alternative conditions. For example, if we subtract equation (4.14) from equation (4.16) we eliminate the constant term and obtain, after cancelling a factor 8 ,

$$
0=16 A-27 A^{2}+10 A^{3}+\left(16-41 A+40 A^{2}-12 A^{3}\right) \alpha
$$

In the same way, we can eliminate the $A^{3} \alpha$ term by adding three times equation (4.14) to equation (4.16). Again, a numerical factor can be cancelled and the result is

$$
0=32-68 A+50 A^{2}-12 A^{3}+\left(16-27 A+10 A^{2}\right) \alpha .
$$

Equations (4.17) and (4.18) can be regarded as an alternative pair to equations (4.14) and (4.16). However, equations (4.17) and (4.18) are related, for if we multiply equation (4.18) by $\alpha$ and use $\alpha^{2}=A-\alpha$ we obtain equation (4.17). Thus we can regard equation ( 4.18 ) as the basic equation for $\alpha$, with equation (4.17) as a derived form and with equations (4.16) and (4.14) as linear combinations of equations (4.17) and (4.18).

This is a satisfactory conclusion since it agrees with the equation $D(\alpha, A)=0$ obtained in Section 3. If we divide $D(\alpha, A)$ by $\alpha^{2}+\alpha-A$ and write the remainder as $R(\alpha, A)$, then

$$
D(\alpha, A)=\left(\alpha^{2}+\alpha-A\right) S(\alpha, A)+R(\alpha, A)
$$

where $S(\alpha, A)$ is a polynomial of degree 16 in $\alpha$ and of degree 8 in $A$ and

(4.20) $\quad R(\alpha, A)=(32768)\left\{\left(32-68 A+50 A^{2}-12 A^{3}\right)+\left(16-27 A+10 A^{2}\right) \alpha\right\}$.

Thus when $C=0$ the equation $D(\alpha, A)=0$ reduces to equation (4.18). 


\section{Numerical results}

Although periodic solutions exist for $A>2$ and the equations obtained in Sections 2,3 and 4 remain valid for $A>2$, the results obtained by Chaundy and Phillips [2] indicate that the periodic solutions are unstable for $A>2$. The numerical working has accordingly been limited to $-1 / 4<A \leq 2$. For $A=2$, Lorenz [3] gives an exact solution in terms of trigonometric functions and these trigonometric solutions were of great help in checking the equations of Sections 2 and 3 . For $C 7$ solutions, the trigonometric solutions are

$$
b_{m}=2 \cos \left(2^{m} \phi\right), m=1,2,3, \ldots, 7,
$$

with

$$
\phi=N \pi / 129 \text { or } \phi=N \pi / 127 \text {, }
$$

for any integer $N$. In practice, all 18 of the $C 7$ solutions were obtained by using in turn $N=1,3,5,7,9,11,13,19,21$. The 18 values of $\alpha$ were compared with the roots of $D(\alpha, 2)=0$ and the agreement was excellent. For any particular solution, combinations of the $b^{\prime}$ 's such as $B_{1}, \gamma_{1}, \beta, \gamma, \delta, \varepsilon, \theta, \zeta$ can be calculated and compared with the values given by the formulae in section 2 .

It is fairly easy to show that, with the above values for $N, \zeta=+1$ when $\phi=N \pi / 127$ and $\zeta=-1$ when $\phi=N \pi / 129$. If $z=\exp (4 i \phi)$ then

$$
\begin{gathered}
1+z=(2 \cos 2 \phi) \exp (2 i \phi)=b_{1} \exp (2 i \phi) \\
1+z^{2}=(2 \cos 4 \phi) \exp (4 i \phi)=b_{2} \exp (4 i \phi) \\
\ldots \ldots \ldots \ldots \ldots \ldots \ldots \ldots \ldots \ldots \ldots \ldots \ldots \ldots \ldots \ldots \ldots \ldots \ldots \ldots \\
\left(1+z^{64}\right)=(2 \cos 128 \phi) \exp (128 i \phi)=b_{7} \exp (128 i \phi) .
\end{gathered}
$$

Also,

$$
\begin{aligned}
1-z & =-2 i(\sin 2 \phi) \exp (2 i \phi), \\
1-z^{128} & =-2 i(\sin 256 \phi) \exp (256 i \phi) .
\end{aligned}
$$

The identity

$$
(1+z)\left(1+z^{2}\right)\left(1+z^{4}\right) \ldots\left(1+z^{64}\right)=\left(1-z^{128}\right) /(1-z),
$$


which is valid for $z \neq 1$, now gives

$$
\zeta \exp (254 i \phi)=\{(\sin 256 \phi) /(\sin 2 \phi)\} \exp (254 i \phi)
$$

and hence

$$
\zeta=(\sin 256 \phi) /(\sin 2 \phi) .
$$

For $\phi=N \pi / 129,256 \phi=2 N \pi-2 \phi$ and $\zeta=-1$. For $\phi=N \pi / 127$, $256 \phi=2 N \pi+2 \phi$ and $\zeta=+1$.

A corresponding result was noted [1] in discussing the $C 6$ solutions for $A=2$ and the proof above can easily be modified to deal with that case. Indeed it appears that for $A=2$ the only cyclic solution which does not have $|\zeta|=1$ is the equilibrium solution $w_{n}=2$.

In looking at the solutions of $D(\alpha, A)=0$ for different values of $A$ it was clear that the real solutions occurred in pairs. For each pair there was a critical value of $A$, denoted by $A^{*}$, at which the pair first appeared. At $A=A^{*}$, there was a double root $\alpha=\alpha^{*}$ and the stability criterion, $\zeta$, equalled $1 / 128$, that is the solution was at the upper limit for local stability. For $A>A^{*}$ the double root split into two distinct roots, with $\zeta<1 / 128$ in one case and $\zeta>1 / 128$ in the other. Thus one family of $\alpha$ values gives unstable solutions while the other gives stable solutions until $\zeta$ becomes less than $-1 / 128$, say for $A^{*}<A<A^{* *}$ where $A^{* *}$ is the value of $A$ at which $\zeta=-1 / 128$. Table 1 lists the critical values $A^{*}$ and $A^{* *}$ for each pair of roots, with the roots numbered in order of increasing magnitude. The table also shows which root in each pair gives stable solutions and, in the last column, values of $b_{i}$ for a typical solution. In each case, the trigonometric solution (for $A=2$ ) corresponding to the stable sequence was used to provide these typical values. It will be seen that there is a marked change in the type of solution as $\alpha^{*}$ increases. A rough figure is given for the interval of stability, $A^{* *}-A^{*}$, and it will be seen that it varies by a factor of about 3000 from the widest to the narrowest interval.

The solutions for the case $A=2$ can be paired off in the same way as the pairs of roots in Table 1 . For example, $\phi=21 \pi / 127$ corresponds to $\alpha_{1}$ and $\phi=21 \pi / 129$ corresponds to $\alpha_{2}$. The values of $\zeta$ for the 


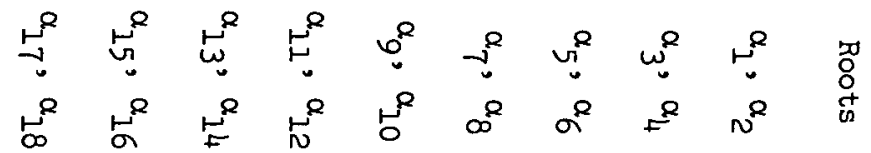

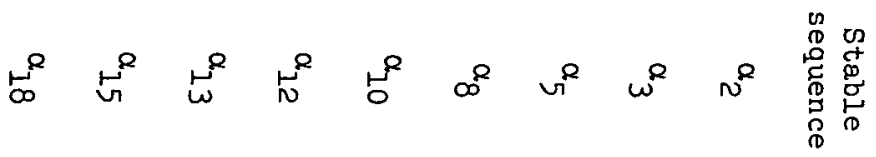

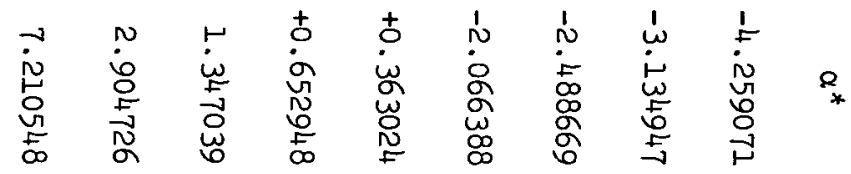

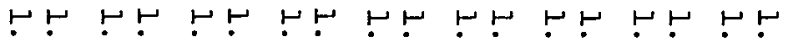
io io io io io io io 它 융요

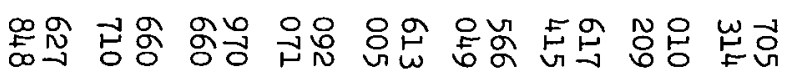

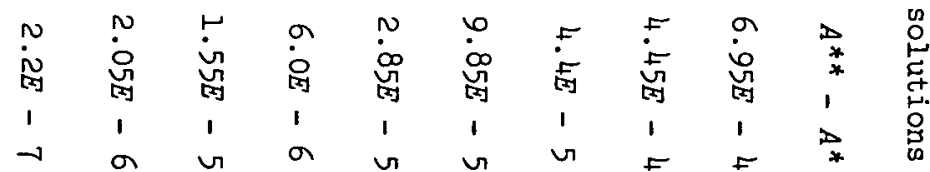

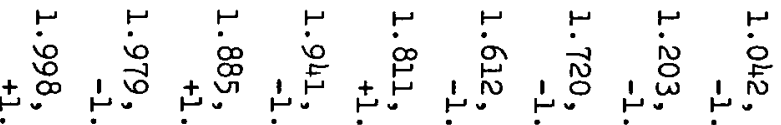

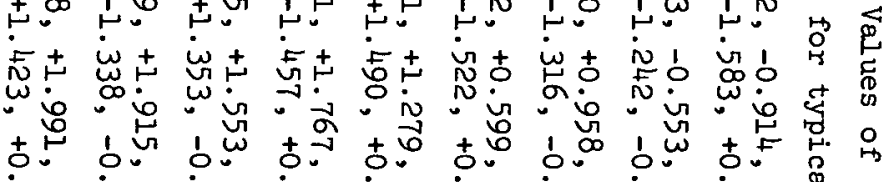

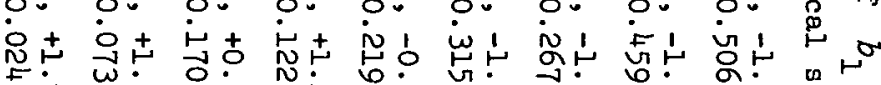
으의

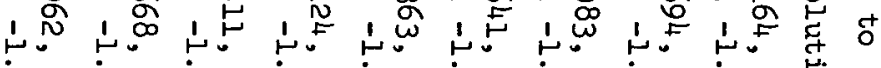

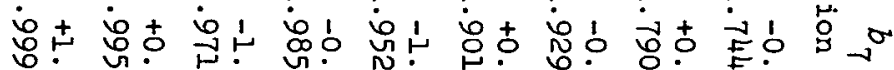

$$
\text { 唄 }
$$


trigonometric solutions provide a check on the identification of the stable family of roots, in the sense that for the $\alpha_{1}$ family $\zeta$ goes from $+1 / 128$ at $A=A^{*}$ to +1 at $A=2$, whereas for the $\alpha_{2}$ family $\zeta$ goes from $1 / 128$ at $A=A^{*}$ to -1 at $A=2$. Thus we can expect the $\alpha_{2}$ family to have $|\zeta|<1 / 128$ for some range of $A$-values. A similar argument applies for the other pairs of roots.

As $A$ increases, the first $C 7$ solution to occur is for $A=1.575$ (which corresponds to $a=1.851$ ). It looks like a perturbation of the equilibrium solution $w_{n}=1-a$ and, as was true for $c 3, c 4, C 5$ and $c 6$ solutions, the smallest value of $\alpha^{*}$ is associated with this smallest value of $A^{*}$. However, there is numerical evidence that this association does not extend to $C 8$ solutions. For the latter, the smallest value of $\alpha^{*}$ appears to arise from a solution which is a perturbation of the equilibrium solution but the smallest value for $A^{*}$ is for a $C 8$ solution which arises when a $C 4$ solution becomes unstable and bifurcates.

For the special case $C=0$, we have

$$
\alpha^{2}+\alpha=A=\alpha^{2}-a
$$

and hence $\alpha=-a$ or $\alpha=a-1$. If we start with a specified value for the constant $a$, with $1 / 2<a \leq 2$, it is easy to calculate $A$ and then

$$
\alpha_{0}=\left(12 A^{3}-50 A^{2}+68 A-32\right) /\left(10 A^{2}-27 A+16\right) .
$$

From equation (4.18), $\alpha_{0}$ is the value of $\alpha$ appropriate to the case $C=0$ and we can compare it with $-a$ and $a-1$. The calculations gave $\alpha_{0}=-a$ for $a=1.9594473$, corresponding to $A=1.879986$, so the equations in Section 4 would be appropriate in this case. The other possibility, $\alpha_{0}=a-1$, did not occur for the range of values tested.

In concluding this section I should like to thank Dr B.L. Martin who carried out the calculations which form the basis of Table 1 and who checked a number of other points numerically. Because of the large powers of $\alpha$ required in calculating $D(\alpha, A)$ double precision was needed in the computer programmes and the values for $A^{*}$ and $A^{* *}$ were obtained to an accuracy of at least $10^{-12}$, although not all of the figures have been 
included in Table 1.

\section{References}

[1] A. Brown, "Equations for periodic solutions of a logistic difference equation", J. Austral. Math. Soc. Ser. B 23 (1981), 78-94.

[2] T.W. Chaundy and Eric Phillips, "The convergence of sequences defined by quadratic recurrence-formulae", Quart. J. Math. Oxford Ser. 7 (1936), 74-80.

[3] Edward N. Lorenz, "The problem of deducing the climate from the governing equations", Tellus 16 (1964), I-Il.

[4] Robert M. May, "Simple mathematical models with very complicated dynamics", Nature 261 (1976), 459-467.

[5] J. Maynard Smith, Mathematical ideas in biology (Cambridge University Press, Cambridge, 1968).

Department of Mathematics,

Faculty of Science,

Australian National University,

PO Box 4,

Canberra,

ACT 2600,

Australia. 